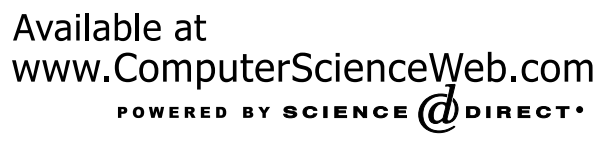

Computer Communications 26 (2003) 1018-1027 computer communications

www.elsevier.com/locate/comcom

\title{
ItswTCM: a new aggregate marker to improve fairness in DiffServ
}

\author{
Hongjun $\mathrm{Su}^{\mathrm{a}}$, Mohammed Atiquzzaman ${ }^{\mathrm{b}, *}$ \\ ${ }^{a}$ School of Computing, Armstrong Atlantic State University, Savannah, GA 31419, USA \\ ${ }^{\mathrm{b}}$ School of Computer Science, University of Oklahoma, Norman, OK 73019-6151, USA \\ Received 19 March 2002; revised 23 August 2002; accepted 10 October 2002
}

\begin{abstract}
Recent demands for real time applications have given rise to a need for Quality of Service (QoS) in the Internet. Differentiated Services (DiffServ) is one of such efforts currently pursued by IETF. Previous researchers found unfairness in the DiffServ network. To solve the unfairness problem, we propose a new TSW based three-color marker (ItswTCM), which achieves proportional fair share of excess bandwidth among aggregates in a DiffServ network. We have compared the fairness of our proposed ItswTCM marker with $s r T C M, \operatorname{trTCM}$, and $t s w T C M$. Results show that our proposed marker performs better than the other three schemes for low to medium network provision level $(20-70 \%)$; we believe that all well provisioned network will operate in this region. Results also show that our proposed marker is not as sensitive to the number of flows in an aggregate as the previous marking schemes. We point out that yellow packets play a significant role in achieving proportional fair share of excess bandwidth among aggregates. We conclude that in order to achieve proportional fair sharing of excess bandwidth, it is important to inject right amount of yellow packets into the network.
\end{abstract}

(C) 2002 Elsevier Science B.V. All rights reserved.

Keywords: TCP protocol; Quality of service; Differentiated services; Traffic Marker

\section{Introduction}

The Internet, based on the TCP protocol, has succeeded in providing world wide data communication service for the past few decades. However, Internet does not provide any Quality of Service (QoS) guarantee to applications. The emerging new service types, such as real-time audio/video applications gives rise to a demand for providing QoS support in the Internet. Differentiated Services (DiffServ) network [1,2] is currently being developed by IETF to provide QoS to applications communicating over the Internet. DiffServ relies on using a Type-of-Service (TOS/ DS) bit in the IP header to provide coarse-grained QoS to applications. Unlike Integrated Service (IntServ) [3], which has scalability problem in the core network, DiffServ avoids the scalability issue by providing QoS guarantees to aggregates rather than individual connections/flows.

Instead of providing guarantees to individual flows, DiffServ provides statistical QoS only to a few predefined service classes. It provides service differentiation among traffic aggregates over a long time scale. A DiffServ network

\footnotetext{
* Corresponding author. Tel.: + 1-405-325-8077; fax: + 1-928-962-8422

E-mail addresses: atiq@ieee.edu (M. Atiquzzaman), atiq@ou.edu (M. Atiquzzaman), hsu@drake.armstrong.edu (H. Su).
}

achieves its service goals by distinguishing between the edge and core network. It pushes all complex tasks, such as traffic classification, traffic monitoring, traffic marking, etc. to the edge network, where per flow based schemes may be used. Traffic passing through the edge network is classified into different service classes, and marked with different drop precedences (such as In/Out packets). As a result, the core routers only need to implement active queue management schemes [4,5], such as RED with In and Out (RIO) [6], and provide service differentiation to the traffic according to preassigned service classes and drop precedences carried in the packet headers. RIO-like active queue management schemes achieve this objective by dropping low priority packets earlier, and with a much higher drop probability, than dropping high priority packets.

Many recent research results, both simulation [7,8,9,22] and mathematical model based [10,11,12], have shown that the current DiffServ proposal has three types of fairness problems: (a) fairness between TCP-friendly traffic and UDP like traffic, (b) fair share of bandwidth among flows in an aggregate, (c) fair share of excess network bandwidth among aggregates. The fairness between TCP and UDP traffic can be alleviated by mapping them into different drop precedences $[13,8]$, by using separate queues, or by using per- flow marking schemes $[8,14]$. The fair share of 
bandwidth among flows in an aggregate can be achieved by using per-flow marking [14]. However, the authors are not aware of any significant effort to solve the problem of fair sharing of excess network bandwidth among aggregates. The objective of this paper is to propose schemes to solve the unfair sharing of excess bandwidth among aggregates.

In this paper, we focus on the fair share of excess bandwidth among aggregates. We first analyze the effect of aggregate based marking schemes on the fair sharing of excess bandwidth among aggregates. We then propose an Improved Time Sliding Window (TSW) based Three Color Marker (ItswTCM). Our proposed scheme is based on the following principles: (a) Protecting well behaved senders (which send traffic according their service contracts); (b) punishing aggressive senders by assigning their traffic to higher drop precedence with high dropping probability, (c) improving the proportional fair share of the excess network bandwidth among aggregates. With proportional fair share, we mean that the excess network bandwidth should be shared among aggregates in proportion to their service profile. We believe this is important for large service subscribers.

Our work differs from previous work in the sense that our new marking scheme improves the proportional fair share of the excess network bandwidth among aggregates. The contributions of this paper are:

- We propose ItswTCM, a new marker which can improve the proportional fair sharing of the excess network bandwidth among aggregates.

- We discuss design issues to address the fairness problems in DiffServ networks.

- Using simulations, we show that ItswTCM can alleviate the unfairness problem among aggregates for a large range of network load, especially for medium load (40$70 \%$ provision level).

- We compare fairness of our marker with other well known marking schemes. Simulation results shown that our new scheme achieves better fairness in most cases.

The rest of the paper is organized as follows. Section 2 gives a brief introduction to some commonly used traffic markers. Section 3 presents our proposed ItswTCM marking scheme. In Section 4, we present simulation results to compare fairness between previous marking schemes and our proposed scheme. Section 6 discusses design issues of fair aggregate based markers, and the effect of parameter settings on our proposed marker. Section 7 concludes this paper.

\section{Traffic markers}

In this section, we give a brief introduction to some commonly used traffic markers which are the most important building blocks of a Diffserv edge router. A marker marks the senders's traffic according to its service profile. Traffic that conforms to the service profile will be marked with low drop precedence, and will receive better service; while non-conforming part of the traffic will be marked with high drop precedence, and will receive a best effort service.

\subsection{Types of traffic markers}

Based on the mechanism used to measure the conformity of the traffic, traffic markers can be classified into two broad categories: token bucket based markers and average rate estimator based markers as described below.

\subsubsection{Token bucket based markers}

Token bucket based markers have been widely studied $[7,15,16,17,12]$. These markers use one or more token buckets to measure the sending traffic. For token bucket based markers, each packet passing through the marker will cost an equivalent amount of tokens. For example, in a simple single token bucket marker, an arriving packet is marked as In-profile if there are tokens in the bucket, otherwise, it will be marked as Out-profile.

To improve the fair share of bandwidth between adaptive and non-adaptive traffic inside an Assured Forwarding (AF) class [13], single rate Three Color Marker (srTCM) and two rate Three Color Marker (trTCM) have been proposed [15, 16]. Two token buckets are used in the three color marking scheme: one holds green tokens, the other holds yellow tokens. When a packet arrives, it is marked as green if both green and yellow tokens are available; it is marked as yellow if only yellow tokens are available, otherwise, it is marked as red $[15,16]$. The number of tokens in the bucket is reduced by the number of tokens used.

\subsubsection{Average rate estimator based markers}

Average rate estimator based markers use average rate estimating algorithm to measure the arrival rate of individual flows or aggregated flows. The most common rate estimating algorithm used in such markers is called TSW algorithm [6]. In this algorithm, the arrival rate is calculated according to the weighted average of the arrival rate over a certain time window/interval. For detailed algorithm, please refer to Ref. [6].

To take advantage of three color marking, TSW based three color marker ( $t s w T C M)$ has been proposed recently [18]. For tswTCM, whenever a packet arrives, the marker calculates the estimated arrival rate. If the estimated arrival rate is less than the Committed Information Rate (CIR), arriving packets are marked as green; otherwise, they are marked as green, yellow or red according to a calculated probability. The $t s w T C M$ algorithm is shown in Fig. 1. We will discuss fairness issues of this marker in Section 2.2. Based on the discussion, we will describe our proposed 
avg-rate $=$ Estimated Avg Traffic Sending Rate

if (avg-rate $<=$ CIR)

the packet is marked as green;

else if (avg-rate $<=$ PIR) AND (avg-rate $>$ CIR)

$$
\text { calculate } \mathrm{PO}=\frac{\text { (avg-rate }-\mathrm{CIR} \text { ) }}{\text { avg-rate }}
$$

with probability $\mathrm{P} 0$ the packet is marked as yellow; else with probability (1-P0) the packet is marked as green;

$$
\begin{aligned}
& \text { calculate } \mathrm{P} 1=\frac{(\text { avg-rate }-\mathrm{PIR})}{\text { avg-rate }} \\
& \text { calculate } \mathrm{P} 2=\frac{(\mathrm{PIR}-\mathrm{CIR})}{\text { avg-rate }}
\end{aligned}
$$

with probability $\mathrm{P} 1$ the packet is marked as red; with probability $\mathrm{P} 2$ the packet is marked as yellow; with probability $(1-(\mathrm{P} 1+\mathrm{P} 2))$ the packet is marked as green;

Fig. 1. Marking algorithm for the tswTCM marker.

tswTCM-based marker, called Improved TSW Three Color Marker ItswTCM, that will improve the fairness of DiffServ.

\subsection{Analysis of unfairness of existing markers}

As thoroughly analyzed in our previous work [12] (and to some extent in Refs. [7-9]), unfairness exists in the sharing of excess network bandwidth among traffic aggregates. It was found in these papers that the excess bandwidth in the core network is shared among flows instead of aggregates, and DiffServ tends to favor small service subscribers. We think there are mainly two reasons for this:

One is due to the TCP congestion algorithm itself. According to current TCP congestion algorithm, small flows will increase their service rate faster than larger flows during the congestion recovery, and therefore can acquire more bandwidth.

The second reason is that existing markers mark the green packets according to their committed information rate $(C I R)$, while the marking of yellow and red packets has little to do with their $C I R$. Green packets correspond to the traffic that commit to their service profiles, and enjoy high priority in the core network; yellow packets have lower priority; and red packets have the lowest priority. While the service rate is ensured by the green packets, the excess network bandwidth at the core network is acquired by yellow or red packets. We believe that marking packets as yellow and red, without considering their service profiles, leads to unfairness among traffic aggregates (i.e. service subscribers).

Let us now look closely at the marking of yellow and red packets in the tswTCM scheme, and discuss its unfairness problem in sharing of excess bandwidth among aggregates. The tswTCM algorithm is shown in Fig. 1. The marking probability of this algorithm is shown in Fig. 2, with PIR being set to 1.5 times of its $C I R$, which plots the marking probability versus avg_rate/CIR, where avg_rate is the average sending rate of an aggregate measured by the TSW algorithm. In this figure, the overall drop precedence is defined as: $d_{1} * p_{\text {green }}+d_{2} * p_{\text {yellow }}+d_{3} * p_{\text {red }}$, where, $d_{1}, d_{2}$ and $d_{3}$ represent three drop precedence levels, and $p_{\text {green }}$, $p_{\text {yellow }}$ and $p_{\text {red }}$ represents the marking probabilities of the Green, Yellow and Red packets, respectively.

For traffic rate less than the $C I R$, all packets are marked as green packets, no packets are marked yellow or red. For traffic rate greater than $C I R$, a portion of the traffic (equal to $C I R$ ) will be marked as green, and the remaining portion of the traffic will be marked as yellow or red according to a calculated probability as given in Fig. 1. Marking CIR part of the traffic as green guarantees the user's service rate. However, unfairness is introduced if the yellow packet marking probability, which we believe plays an important role in achieving fair share of excess bandwidth, is not proportional to their CIRs. This is indeed the case for this algorithm as we will see shortly.

For $a v g \_$rate $>P I R$, increase in the sending rate will decrease the marking probability of yellow packets, whereas the probability will increase for red packets. This will indeed help to punish the aggressive sender.

The main problem associated with this algorithm is in $C I R<a v g$ rate $\leq P I R$. Note that in this region, the yellow packet marking probability is proportional to the excess bandwidth, i.e. avg_rate - CIR, acquired by this aggregate rather than the service rate $(C I R)$ of this aggregate. This marking behavior introduces a serious unfairness problem. Because this algorithm says that "If you can acquire more excess bandwidth, I will mark more of your packet as yellow!", it encourages the aggressive senders in this region! The more excess bandwidth you can grab, the more your packet will be marked as yellow. Small service subscribers or an aggregate with more flows usually benefit in this region due to the inherent unfairness of the TCP congestion algorithm. Large service subscribers, although paying more money for the service, have no way to compete with the smaller ones for excess bandwidth in this region.

\section{ItswTCM: the proposed marker to ensure fairness}

In this section, we list the notations used in this paper, analyze the shortcomings of previous marking algorithms with respect to fairness, and then present our proposed marking algorithm along with its advantages over previous algorithms.

\subsection{Notations}

MSS TCP's Maximum Segment Size.

RTT Round Trip Time of a TCP connection.

CIR Committed Information Rate, the contracted average sending rate. In $s r T C M$ and $\operatorname{trTCM}$, it is used as the arrival rate of the green tokens.

PIR Peak Information Rate, the maximum contracted sending rate. In $\operatorname{trTCM}$ scheme, it is used as the arrival rate of yellow tokens. 


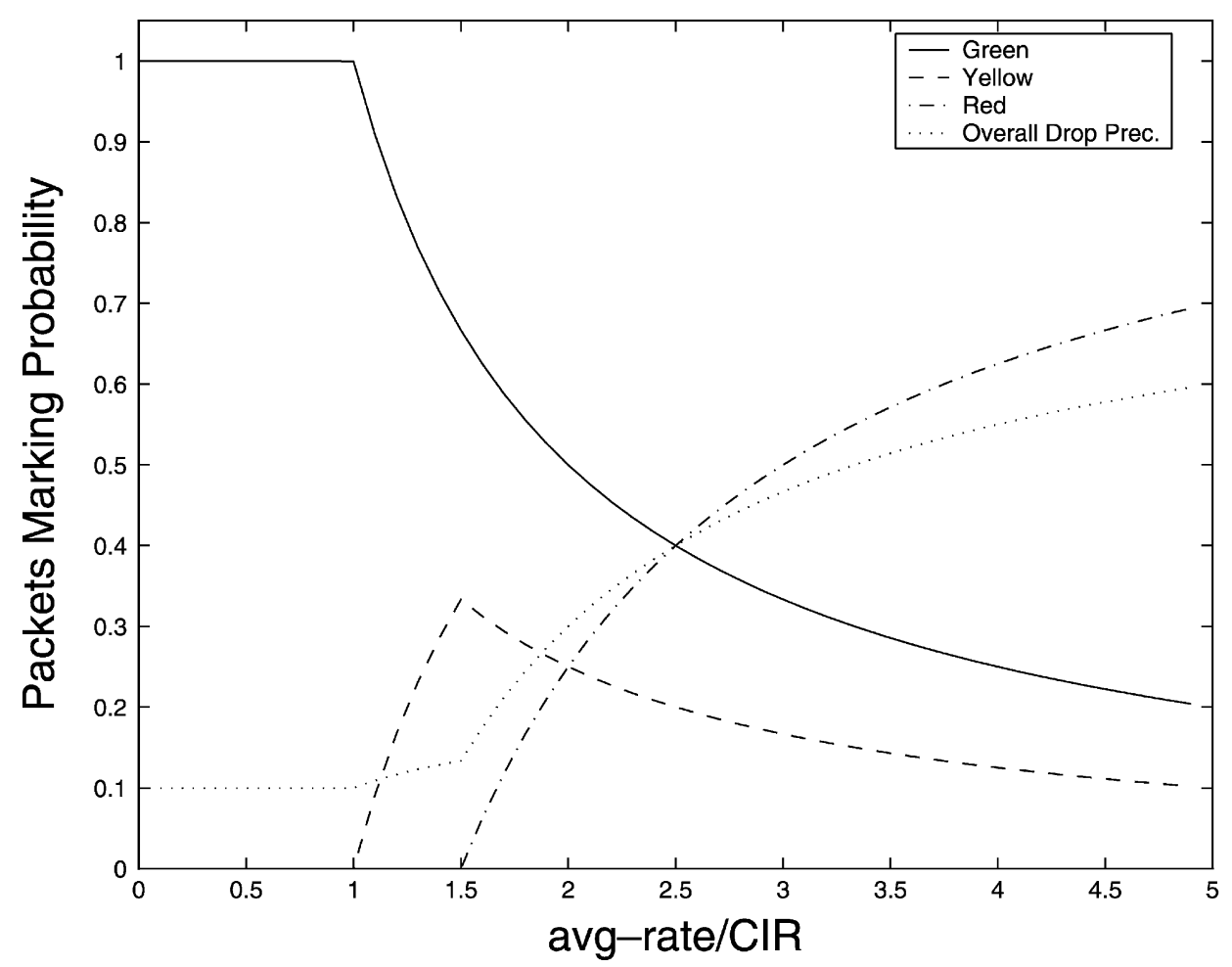

Fig. 2. Marking probability and overall drop precedence of the tswTCM algorithm.

CBS Committed Burst Size, the contracted traffic burst size. In $\operatorname{srTCM}$ and $\operatorname{trTCM}$, it is used as the green token bucket size.

EBS Excess Burst Size, the excess token bucket (used to hold excess tokens) size in the srTCM scheme.

PBS Peak Burst Size, the yellow token bucket size in the trTCM scheme.

\subsection{Algorithm of ItswTCM}

To alleviate the problem of unfair sharing of the excess bandwidth among aggregates (see Section 2.2), we propose the Improved TSW based Three Color Marker scheme (ItswTCM) as shown in Fig. 3. The basic idea is to allow an aggregate to inject yellow packets in proportion to its $C I R$. This means that a large service subscriber will be able to inject more yellow packets than a small service subscriber. ItswTCM can therefore, improve the inherent unfairness of TCP congestion algorithm, and result in better fair share of excess bandwidth.

In ItswTCM, for avg_rate $<C I R$, all packets are marked as green packets (this is same as the previous algorithm), which guarantees the service rate. The main difference between our proposed algorithm and the previous algorithm is in the region $a v g \_r a t e>C I R$ : Unlike the previous algorithm, once its average rate exceeds its $C I R$, no packet in an aggregate will be marked as green by our proposed algorithm.

In our algorithm, the region avg_rate $>C I R$ is divided into two subregions:
1. For $C I R<a v g \_r a t e<\mathrm{c}^{*} C I R$, all packets from an aggregate are marked as yellow. The reason to introduce this region is to alleviate the unfairness of TCP congestion control algorithms, and allow large flows to have a better chance to compete with small ones.

2. For $a v g \_$rate $>c^{*} C I R$, packets in an aggregate are marked as yellow with probability $\left(c^{*} C I R / a v g \_r a t e\right)^{2}$, and the rest of the traffic in that aggregate are marked as red.

Note that the yellow packet marking probability of an aggregate is proportional to its service rate $(C I R)$ in our algorithm. This helps to achieve better proportional fair sharing of excess bandwidth. The quadratic term in the probability calculation helps to punish aggressive senders once their rates exceed $c^{*} C I R$. The marking probability of our proposed scheme is shown in Fig. 4 for $c=1: 8$. It is

\footnotetext{
avg-rate $=$ Estimated Avg Traffic Sending Rate

$\mathrm{c}=\mathrm{a}$ constant $(\mathrm{c}>1)$

if (avg-rate $<=$ CIR)

the packet is marked as green;

else if (avg-rate $<=c^{*}$ CIR)

the packet is marked as yellow;

else

calculate $\mathrm{P}=\left(\frac{\mathrm{c}^{*} \mathrm{CIR}}{\text { avg-rate }}\right)^{2}$

with probability $\mathbf{P}$ the packet is marked as yellow; with probability 1-P the packet is marked as red;
}

Fig. 3. Marking algorithm of the ItswTCM marker. 


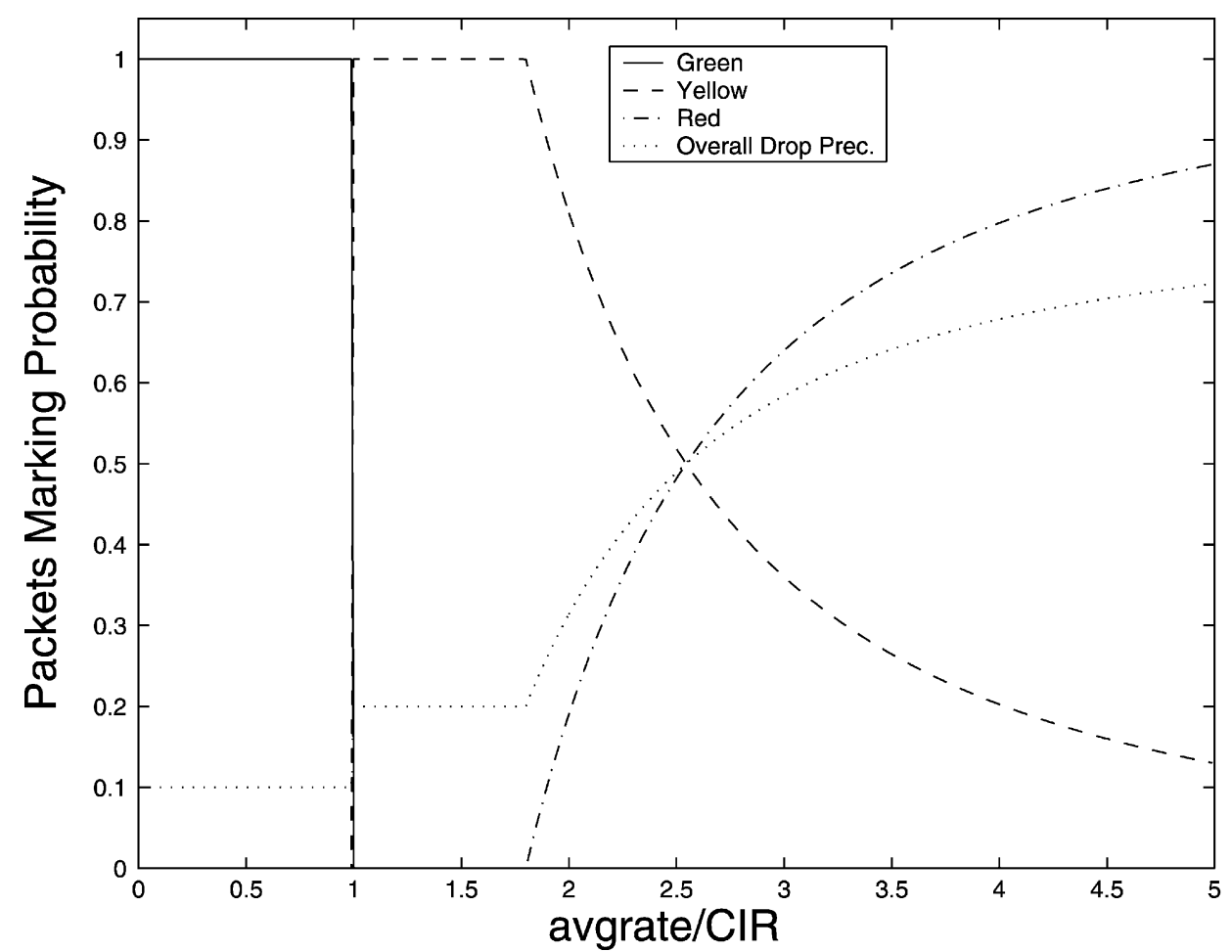

Fig. 4. Marking probability and overall drop precedence of the ItswTCM algorithm.

seen that, in contrast to previous algorithms, yellow packets are marked in proportion to their CIR.

As we pointed out in Section 2.1, existing marking algorithms always mark the CIR portion of packets from an aggregate as green in order to guarantee the subscriber's service rate. However, in our proposed marker, no packet from an aggregate is marked as green when the rate of that aggregate exceeds its $C I R$. A natural question to answer is: Will the service rate of a subscriber be guaranteed in ItswTCM? The answer is "yes". For avg_rate $<C I R$, i.e. when the network is overloaded, there is no difference between the proposed and existing algorithms; for avg_rate $>C I R$, the service rate is obviously achieved. The only difference in this region is deciding on which packet helps to guarantee the service rate: green packet or yellow packet? In the existing algorithm, the service rate is achieved mainly by green packets, while in ItswTCM, the service rate is achieved by yellow packed in this region. Note that avg_rate $>C I R$ implies that the network has excess bandwidth, or there is no serious congestion in the network. Therefore, it is not very critical whether the throughput is realized by green or yellow packets. Furthermore, ItswTCM has the following benefits over existing markers:

- It provides a strong incentive for sources to obey their service contracts. If they exceed their service rates, all their traffic will be marked as yellow or red (no more green traffic as in existing algorithms !!!). Senders have to face possible service quality degradation, such as expecting higher delay.
- It will help to protect TCP-friendly traffic from UDPlike traffic. In ItswTCM, all UDP-like packets are marked as yellow if they do not obey their corresponding traffic profiles. UDP-like traffic may, therefore, suffer higher drop probability in the case of network congestion. TCP-friendly sources can respond to congestion by reducing their rates, and achieve their desired service rate by having all their packets marked as green.

\section{Simulation setup and performance criteria}

In this section, we describe the simulation topology and performance criteria that we have used in Section 5 to compare the fairness of existing schemes and ItswTCM. Simulations have been performed using the ns-2 network simulator [19], together with the DiffServ patch from Nortel [20].

\subsection{Simulation setup}

Fig. 5 shows the simulation topology used in this study. There are two groups of sources, called aggregate 0 and aggregate 1. Aggregate 0 sends traffic through the edge router E0 to the destination D0, while aggregate 1 sends traffic through the edge router E1 to the destination D1. Each aggregate contains a number of flows from different source nodes $(\mathrm{S})$. E0 and E1 are the edge routers responsible for monitoring and marking traffic aggregates 0 and 1, respectively. 


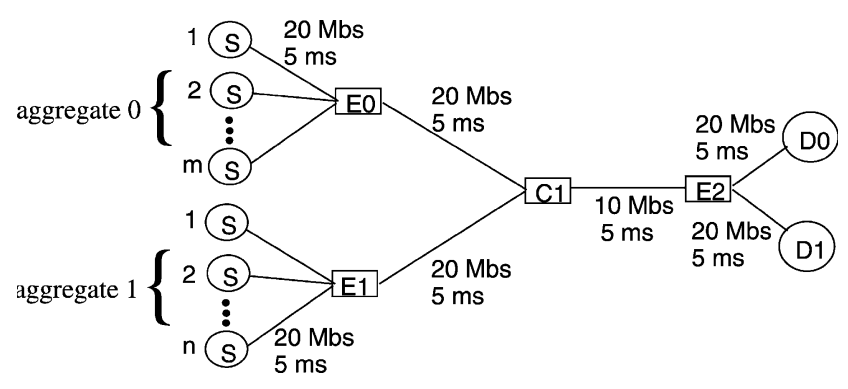

Fig. 5. Simulation topology.

$\mathrm{C} 1$ is the core router that implements active queue management, and provides service differentiation among aggregates according to the drop precedences carried in the packet headers. In this simulation, we use the multi-RED queue management scheme [4]. We use the notation $\{x, y, z\}$ to represent minimum threshold, maximum threshold, and weight parameter, respectively, of the RED queue [4]. The general guidelines as given in Refs. [4,6] is followed in setting the RED parameters. The settings of the core router is $\{0,40,0.2\},\{40,80,0.1\}$ and $\{80,120,0.02\}$ for Red, Yellow and Green packets, respectively. We use TCP Reno in all the simulations, with all TCP flows being long lived FTP applications with $M S S=1500$ bytes and $R T T=40 \mathrm{~ms}$ for both aggregates.

For $\operatorname{srTCM}$ and $\operatorname{trTCM}$, token arrival rates are set to their CIRs. CBS is set to 30000 bytes or 20 packets. For srTCM, $E B S$ is set to 36000 bytes. For $\operatorname{trTCM}, P B S$ is set to 36000 bytes, while $P I R$ is set to $1.2 \times C I R$. The PIR for $t s w T C M$ is also set to $1.2 \times C I R$. These settings simulate the case where a source has negotiated an average sending rate of $C I R$, with its peak sending rate of at most $120 \%$ of its CIR. The authors realize that different settings may have different effects on the schemes. However, choosing the optimum setting for the algorithms is out of scope of this paper. For ItswTCM, we set $c=2$; the effect of $c$ will be discussed in Section 6 .

\subsection{Fairness criteria}

To compare the fairness between our proposed scheme and existing schemes, we used the most commonly used definition of Fairness Index (FI) [21] as given below:

$F I=\frac{\left(\Sigma_{i} x_{i}\right)^{2}}{N^{*} \Sigma_{i} x_{i}^{2}}$

where, $0<$ FI $<1$,

$x_{i}=\frac{\text { excess bandwidth obtained by aggregate } i}{C I R \text { of aggregate } i}$

and $N$ is the total number of aggregates under consideration (in our case, $N=2$ ). According to this definition, the closer the fairness index is to one, the fairer is the distribution of the excess bandwidth between the aggregates. Note that, we use proportional fair share rather than equal fair share.

\section{Results}

In this section, we compare the fairness of our proposed (ItswTCM) scheme with those of three existing marking schemes: Single Rate Three Color Marker (srTCM) [16], two rate Three Color Marker(trTCM) [15], and TSW based Three Color Marker (tswTCM) [18].

We studied the fairness of four marking schemes for three different cases. In all the cases, the $C I R$ for aggregate 1 was fixed at $1 \mathrm{Mbps}$, while the $C I R$ for aggregate 0 increases from 1 to $8 \mathrm{Mbps}$. This corresponds to the network going from a provision level of 20-90\%. We define the provision level to be the ratio of total provisioned bandwidth to the bandwidth of the bottleneck link, which is $10 \mathrm{Mbps}$ in our case.

We did not consider the overloaded case, because in this case, ItswTCM marks all the packets as green, which is the same as previous schemes as mentioned in Section 3.2. The algorithms should behave similarly under the overloaded condition; the comparisons under the overloaded condition are, therefore, omitted from the discussion.

\subsection{Case 1: aggregates 0 and 1 have 16 flows each}

In this case, aggregates 0 and 1 have 16 flows each. This scenario illustrates how a small service subscriber and a larger service subscriber, emitting the same number of flows, compete for excess bandwidth.

Fig. 6 shows the fairness index versus provision level for four marking schemes. We see that our ItswTCM performs significantly better than others for a large range of provision level, with trTCM and tswTCM performing slightly better than $s r T C M$. Note that at a high provision level (let us say above 75\%), our scheme is worse than $\operatorname{trTCM}$ and $t s w T C M$. The main reason for this performance degradation of our scheme in this region is that almost all the packets from both aggregates are marked as yellow at this load, with almost no green or red packets. The result is that the network is back to a best effort network, with no service differentiation, and TCP congestion control algorithm is in total control. In this case, the effect of dropping yellow packets is more serious for a large service subscriber than for a small subscriber. It is a fact that TCP congestion control algorithms favor small flows [12,7,8,9] which leads to the performance degradation in this region. This also applies to the other two cases discussed in Sections 5.2 and 5.3 .

\subsection{Case 2: aggregates 0 and 1 have 16 and 32 flows respectively}

In this case, aggregates 0 and 1 have 16 and 32 flows, respectively. This case illustrates the sharing of excess bandwidth between a small service subscriber emitting a large number of ows and a larger service subscriber emitting few flows. 


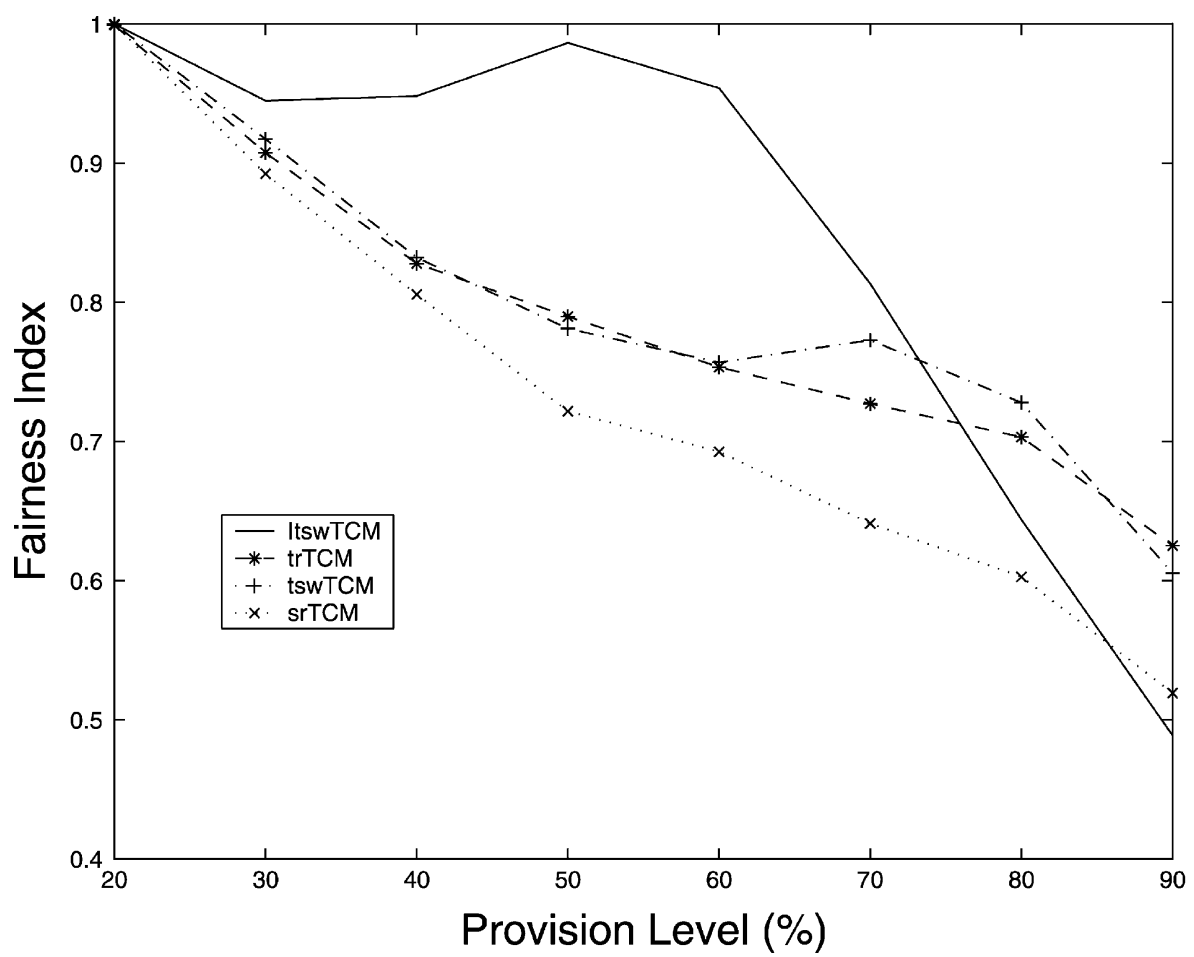

Fig. 6. Fairness Index versus provision level, for aggregate 0(16 flows) and aggregate 1(16 flows) case.

Fig. 7 shows the fairness index versus provision level for the four marking schemes. Again, our proposed marking scheme achieves better fair share of the excess bandwidth than other schemes for a large range of provision level, with the worst one being $s r T C M$.
5.3. Case 3: aggregates 0 and 1 have 32 and 16 flows respectively

In this case, aggregates 0 and 1 have 32 and 16 flows, respectively. This case corresponds to the sharing of

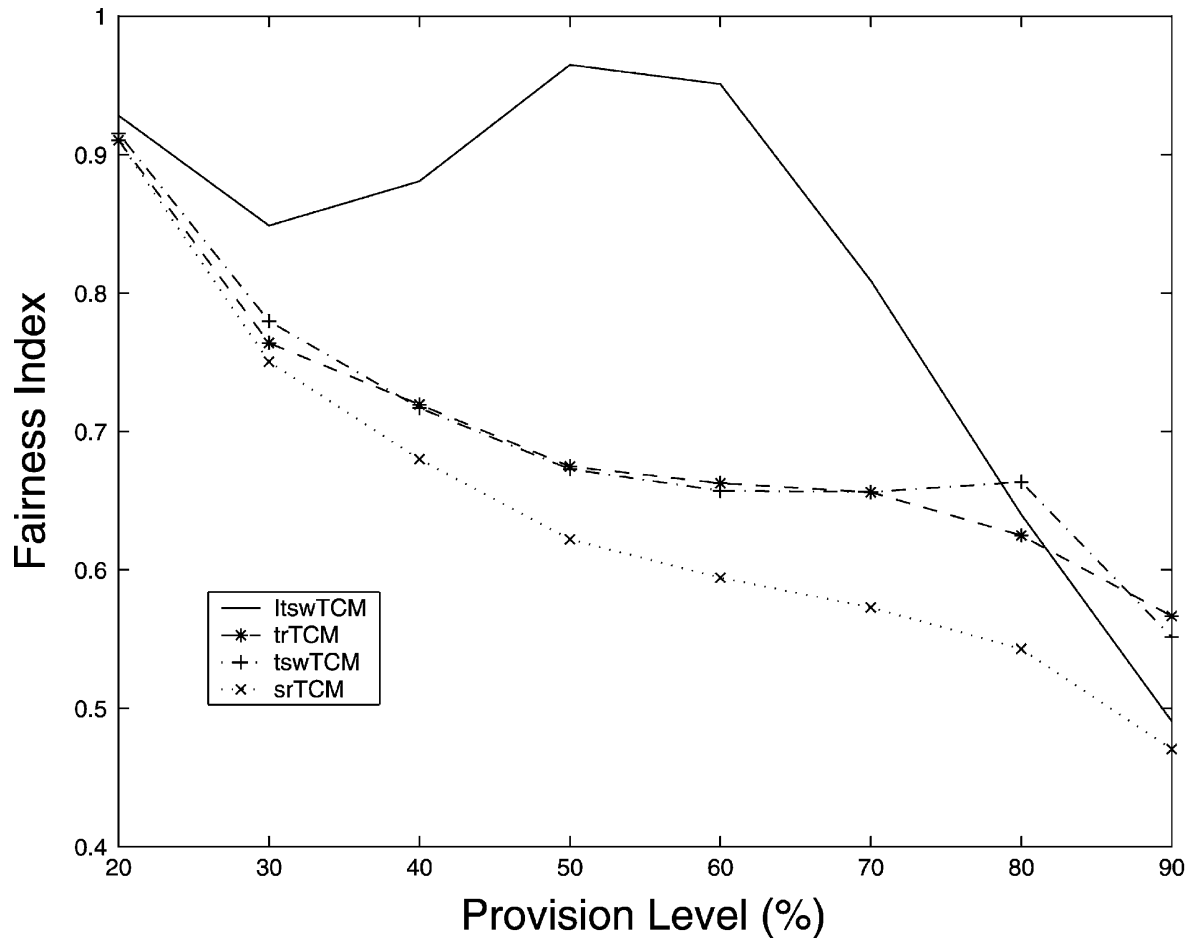

Fig. 7. Fairness Index versus provision level, for aggregate 0 (16 flows) and aggregate 1(32 flows) case. 


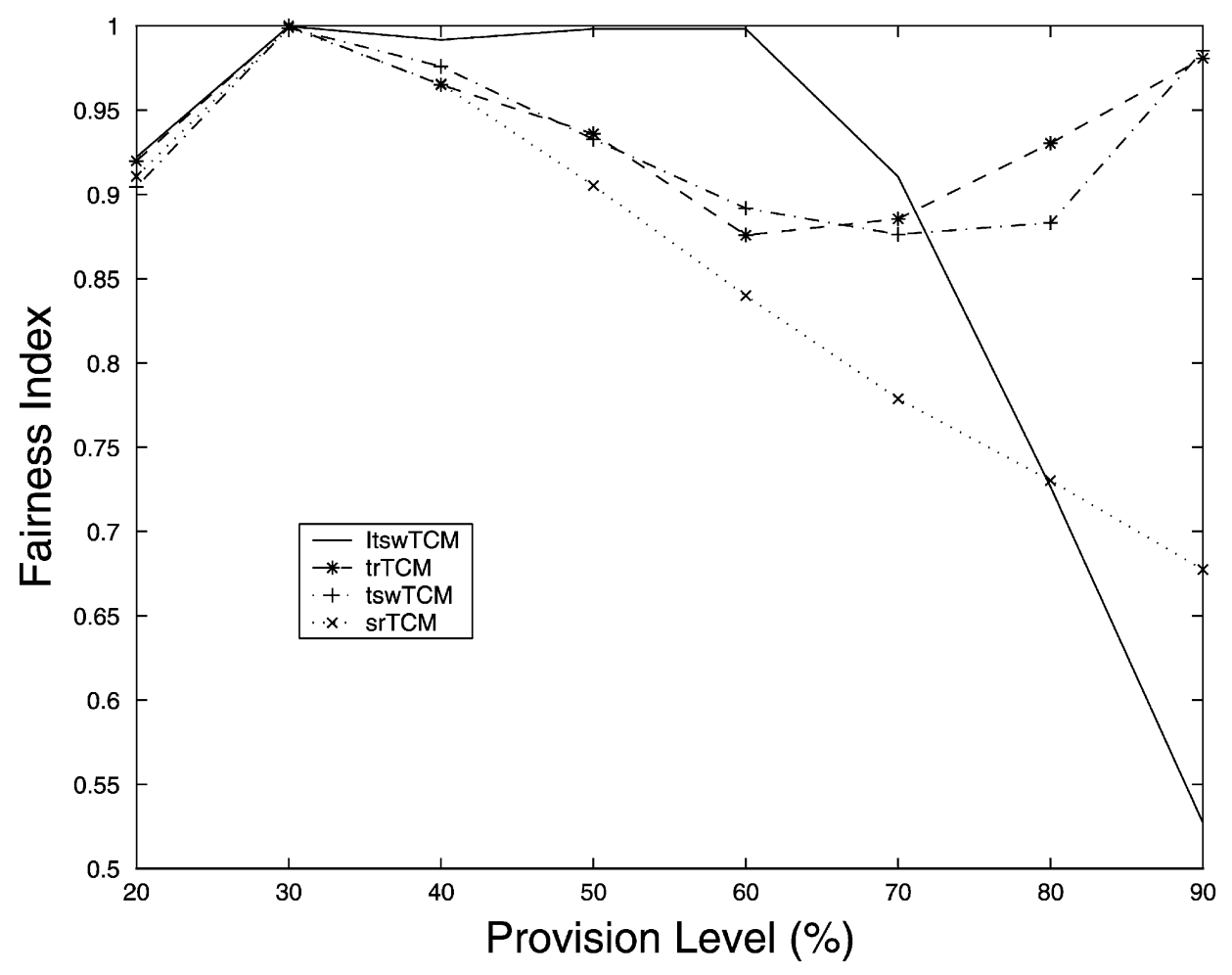

Fig. 8. Fairness Index versus provision level, for aggregate 0 (32 flows) and aggregate 1(16 flows) case.

excess bandwidth between a small service subscriber emitting a small number of flows and a large service subscriber emitting a large number of flows.

Fig. 8 shows the fairness index versus provision level for the four marking schemes. It is seen that our scheme again performs better than other schemes in the 20-70\% provision level. srTCM is still the worst choice. All schemes performs better in this case than their corresponding ones given in Sections 5.1 and 5.2. This is because the larger profile aggregate 0 has more flows, and therefore, it is easier to achieve the proportional share of the excess bandwidth. As in Case 1, previous schemes perform better than our proposed scheme for a high provision level(says between 75 and $100 \%$ ). For overload condition (more than 100\%), these algorithms will all have the similar behaviors because they all mark almost all packets as green, as mentioned in Section 3.2. However, it is expected that a typical network will be provisioned below $80 \%$ provision level, in which case, our scheme is better than all previous schemes in all ranges.

\section{Discussion}

The results presented in Section 4 show that ItswTCM achieves better fairness than other schemes for low to medium level (say from 20 to $75 \%$ ) of provision level. By comparing Figs. 6-8, we see that the shape of the fairness curve for our proposed scheme does not change much, while the curves change significantly for the other three schemes. This means that ItswTCM is not as sensitive as other schemes to a change in the number of flows per aggregate; the performance of our proposed scheme is mainly aggregate based.

Fig. 9 shows the performance of ItswTCM for different values of $c$. In this simulation, the worst of our three case is used, i.e. aggregate 0 and 1 has 16 and 32 flows, respectively. In this case, aggregate 1, which only has a subscribed rate of $1 \mathrm{Mbps}$, contains 32 flows and therefore behaves more aggregate. It is clear that our proposed scheme still cannot achieve proportional fair share of excess bandwidth over the whole region for any of the values of $c$. However, it achieves better performance in terms of fair share in selected regions (depending on value of $c$ ). Except for $c=1$, we see that as $c$ decreases, the fair share region moves from low provision level toward high provision level. It is interesting to note that the maximum value of the fairness index in the region decreases as it moves toward high provision level. This is because, the packet drop probability at high provision level is significantly higher than in the low provision level. Because it requires a significant amount of time for the large profile aggregate to recover its sending rate following a packet loss, the effect of dropping a packet is much more serious for large profile aggregates than for small profile aggregates.

It is interesting to have a closer look for $c=1$, where the performance of ItswTCM is similar to the worst scheme, srTCM. From the marking probability in Fig. 4 and the ItswTCM algorithm in Fig. 3, we see that for $c=1$, the overall probability of marking a packet as yellow is small, i.e. most of the traffic will be marked as either green or red. 


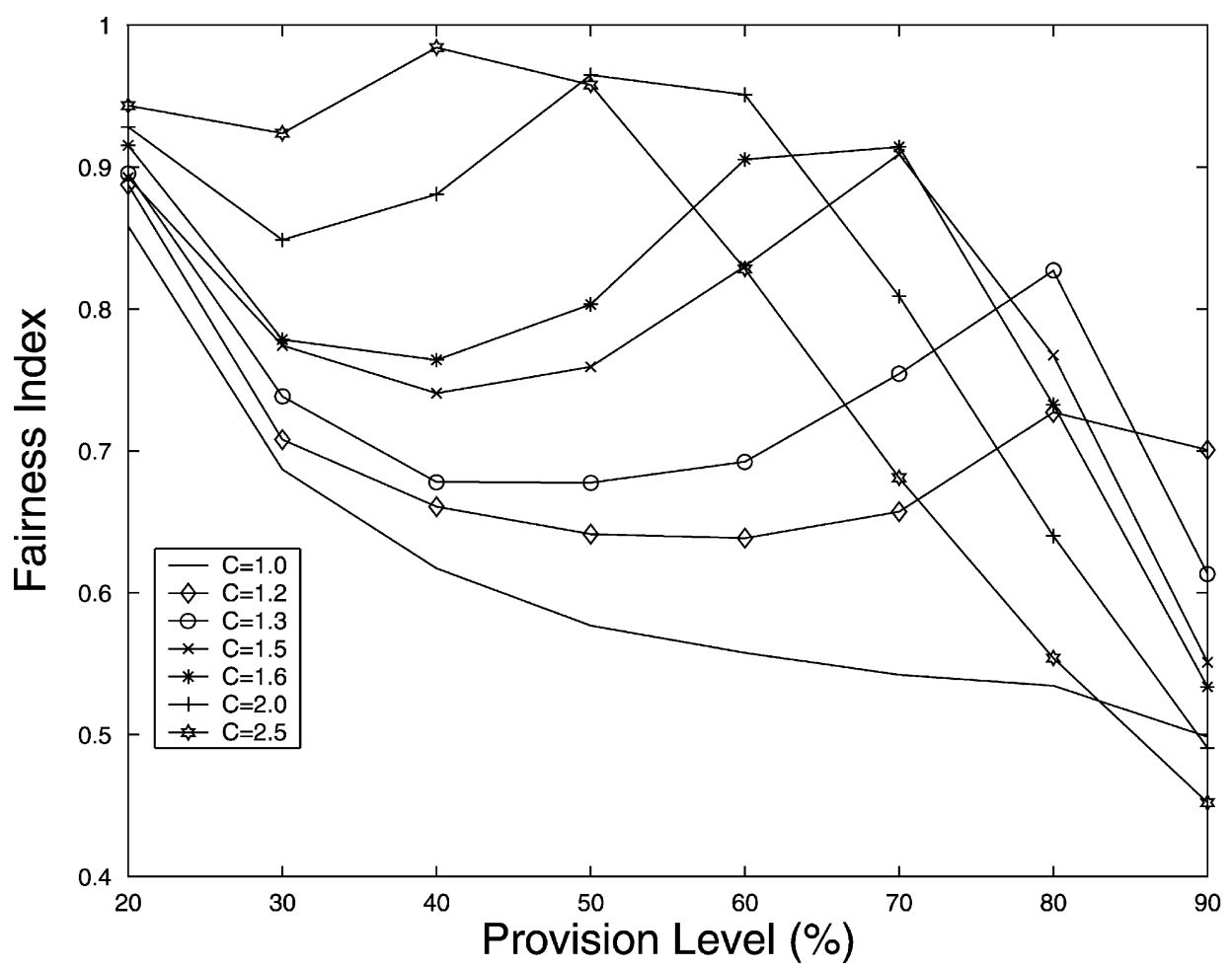

Fig. 9. Effect of factor $c$ to ItswTCM.

This result verifies our earlier observation, i.e. yellow packets play a very important role in realizing proportional fair share of excess bandwidth. This also explains why the tswTCM scheme performs poorly with respect to fairness. From Fig. 2, we see that for the tswTCM scheme, the overall yellow packet marking probability is also small, and therefore performs bad in terms of fairness.

\section{Conclusions}

In this paper, we proposed a new aggregate based marker (ItswTCM) to improve the fairness in the proportional share of excess bandwidth among aggregates in a DiffServ network. The results from this work can be summarized as follows:

- We proposed a new TSW based three color marker, ItswTCM. It is an aggregate marker which is designed to achieve proportional fair share of the excess bandwidth among aggregates in a DiffServ network.

- We compared the fairness of our proposed ItswTCM marker with srTCM, trTCM, and tswTCM. Simulation results show that our proposed marker performs better than the other three markers for low to medium network provision level (20-70\%). We believe that, to avoid any network congestion, all well provisioned networks will operate in this provision level.

- For different network conditions, we found that ItswTCM is not as sensitive to the number offlows in an aggregate as the other three markers. Our scheme is mainly aggregate based.

- By studying the effect of parameter settings on the performance of our marker, and the marking probability of our proposed marker, we found that yellow packets play a significant role in achieving proportional fair share of excess bandwidth among aggregates in a DiffServ network. We concluded that in order to achieve proportional fair share of excess bandwidth, it is important to inject the right amount of yellow traffic into the network.

\section{References}

[1] S. Blake, D. Black, M. Carlson, E. Davies, Z. Wang, W. Weiss, An architecture for differentiated services, RFC 2475 December (1998).

[2] K. Nichols, V. Jacobson, L. Zhang, A two-bit differentiated services architecture for the internet, RFC 2638, Internet Engineering Task Force July (1999).

[3] R. Braden, D. Clark, S. Shenker, Integrated services in the internet architecture: an overview, RFC 1633 June (1994).

[4] S. Floyd, V. Jacobson, Random early detection gateways for congestion avoidance, ACM/IEEE Transactions on Networking 1 (4) (1993) 397-413.

[5] B. Braden, D. Clark, J. Crowcroft, S. Deering, D. Estrin, S. Floyd, V. Jacobason, G. Minshall, C. Partridge, L. Peterson, K. Ramakrishnan, S. Shenker, J. Wroclawski, L. Zhang, Recommendations on queue management and congestion avoidance in the internet, RFC 2309, Internet Engineering Task Force April (1998).

[6] D. Clark, W. Fang, Explicit allocation of best effort packet delivery 
service, IEEE/ACM Transactions on Networking 6 (4) (1998) $362-373$.

[7] J. Ibanez, K. Nichols, Preliminary simulation evalustion of an assured service. draft-ibanez-diffserv-assured-eval-00.txt, Internet Draft, August 1998.

[8] B. Nandy, N. Seddigh, P. Pieda, J. Ethridge, Intelligent traffic conditioners for assured forwarding based differentiated services networks, IFIP High Performance Networking (HPN Paris, France, June (2000).

[9] I. Yeom, A.L. Narasimha Reddy, Realizing throughput guarantees in a differentiated services network, Proceedings of ICMCS 2 (June 7-11) (1999) 372-376.

[10] I. Yeom, A.L. Narasimha Reddy, Modeling TCP behavior in a differentiated services network, Texas A\&M Technical Report May (1999).

[11] M. Baines, B. Nandy, P. Pieda, N. Seddigh, M. Devetsikiotis, Using TCP models to understand bandwidth assurance in a differentiated services network, Nortel Technical Report July (2000).

[12] H. Su, M. Atiquzzaman, Performance modeling of differentiated service network with a token bucket marker, 11th IEEE Workshop on Local and Metropolitan Area Networks, Boulder CO., USA March 18-21 (2001) 81-82.

[13] J. Heinanen, F. Blaker, W. Weiss, J. Wroclawski, Assured forwording PHB group, RFC 2597, Internet Engineering Task Force June (1999).
[14] I. Yeom, A.L. Narasimha Reddy, Impact of marking strategy on aggregated flows in a differentiated-services network, International Workshop on QOS, Landon, UK May (1999) 156-158.

[15] J. Heinanen, R. Guerin, A two rate three color marker, RFC 2698 September (1999)

[16] J. Heinanen, R. Guerin, A single rate three color marker, RFC 2697 September (1999)

[17] S. Sahu, P. Nain, D. Towsley, C. Diot, V. Firoiu, On achievable service differentiation with token bucket marking for TCP, Technical Report 99-72, University of Massachusetts, Deptartment of Computer Science, 1999

[18] W. Fang, N. Seddigh, B. Nandy, A time sliding window three colour marker (tswtcm), RFC 2859 June (2000).

[19] Network simulator 2 (ns-2). University of California at Berkeley, CA, Available via http://www.isi.edu/nsnam/ns/, 1997.

[20] Diffserv model for the ns 2 simulator. Open IP Group at Nort el, available from http://www7.nortel.com:8080/CTL/.

[21] R. Jain, The Art of Computer Systems Performance Analysis: Techniques for Experimental Design, Measurement, Simulation, and Modeling, April, Wiley, New York, NY, 1991, p. 36 and 387.

[22] H. Su and M. Atiquzzaman, "Performance analysis of differentiated services network with token bucket marker", 11th IEEE Workshop on Local and Metropolitan Area Networks, March 18-21, 2001, Boulder, Co, USA, 81-82. 